\title{
Stimulus control of foodcup approach following fixed ratio reinforcement*
}

\author{
RICHARD B. DAY and JOHN R. PLATT \\ McMaster University, Hamilton, Ontario, Canada
}

\begin{abstract}
Rats were trained on fixed ratio (FR) schedules requiring either 5 or 10 leverpresses to produce reinforcement and an intertrial interval (ITI). Half of the Ss at each ratio requirement were extinguished on an FR 5 and half on an FR 10 schedule of ITI presentation. Fewer foodcup approaches were made on the FR 10 than on the FR 5 extinction schedule, regardless of acquisition FR. Leverpresses per approach were fewer on the FR 5 than on the FR 10 extinction schedule and were fewer following FR 5 than following FR 10 acquisition. Data suggested the existence of interoceptive as well as exteroceptive stimulus control of foodcup approach and were discussed in terms of their implications for a response-unit account of extinction.
\end{abstract}

According to Skinner's (1938) response-unit hypothesis, the increased resistance to extinction often shown by intermittently as compared to continuously reinforced responses stems from the fact that intermittent reinforcement strengthens the behavior of emitting groups of responses, while continuous reinforcement strengthens the individual responses. Expanding on this hypothesis, Mowrer and Jones (1945) suggested that resistance to extinction, if measured in terms of the appropriate response unit, should be equal following both intermittent and continuous reinforcement. In the case of fixed ratio (FR) reinforcement, they argued, the reinforced response unit is the entire ratio. Ss trained under different ratio requirements should complete the same number of ratios in extinction while emitting a total number of responses which varies directly with the ratio requirement.

Mowrer and Jones tested this hypothesis by extinguishing a leverpressing response in rats following training on several values of a modified FR schedule of reinforcement. Their schedule, which has since been termed "fixed number" (FN), required a response in addition to leverpressing to produce reinforcement. Specifically, food was delivered when the $S$ approached the food tray following completion of the leverpress requirement. Tray approaches made prior to completion of the leverpress requirement had no programmed consequences. This modification was designed to prevent exteroceptive stimulus events (e.g., magazine operation) from serving as cues of ratio completion. Ss trained to approach the food tray only following food delivery might, for example, emit longer response sequences in extinction while awaiting presentation of these discontinued exteroceptive stimuli. Mowrer and Jones found that the number of ratios completed in extinction was not constant across ratio size, as the response-unit hypothesis predicted, but rather decreased with

*This research was supported by National Research Council of Canada Grant A8269. increasing ratio size. Similar results have been obtained with FR schedules by Boren (1961) and by Weiss (1961). Mowrer and Jones attributed the decreased resistance to extinction shown by longer response units to the increased effortfulness of such units.

Results in accord with a response-unit hypothesis were obtained by Denny, Wells, and Maatsch (1957), who argued that ratio size was not an adequate measure of response-unit length. According to their discrimination model of FR performance, response-unit size is indicated by the number of individual responses which precede some heterogeneous terminal response (e.g., food tray approach) that bounds successive sequences of responses. The correspondence between this response unit and an FR requirement depends upon the extent to which the terminal response comes under the discriminative control of stimuli, such as magazine operation, which signal ratio completion. To the extent that this discrimination is incomplete, response-unit size and ratio requirement will differ.

According to the Denny et al model, decrements in the strength of the acquired response unit occur when terminal responses are nonreinforced during extinction. The number of such terminal responses emitted during extinction should be independent of response-unit length, while the number of individual responses emitted should be a function of the length of the acquired response unit. Denny et al examined extinction following continuous and FR reinforcement of a leverpressing response in rats. Their data revealed that the number of leverpresses emitted in extinction was greater following FR 5 than following continuous reinforcement, while the number of terminal responses (i.e., food tray approaches) was the same for both groups.

Both the model and the data presented by Denny et al suggest the crucial role of terminal responses and their controlling stimuli in the development and extinction of a response unit. In acquisition, the response unit is strengthened each time its termination is followed by 
reinforcement. In extinction, each nonreinforced terminal response reduces the strength of the response unit. The finding that the number of terminal responses emitted in extinction is independent of response-unit length also indicates that increased resistance to extinction following partial reinforcement must be a function of differences in response-unit length during extinction. It is the assumption of any response-unit hypothesis that differences in response-unit lengths during extinction reflect differences in the length of the response unit that was strengthened during acquisition. According to Denny et al, this strengthening involves a stimulus discrimination process such that terminal responses eventually occur only to stimuli which signal completion of the ratio requirement. Maintenance of response-unit lengths in extinction presumably involves both availability of these stimuli and their continued control over terminal responding. Denny et al suggested that this stimulus function is usually served by auditory stimuli associated with food delivery, and their own extinction procedure involved the presentation of a food magazine click following each ratio completion. The role of such exteroceptive stimuli in maintaining response-unit length during extinction is of considerable theoretical importance to any response-unit formulation of extinction performance. If exteroceptive stimuli alone control terminal responding, response-unit length in extinction should be a function of the schedule of exteroceptive stimulus presentation in extinction, rather than of acquisition response-unit length. The correspondence between acquisition and extinction response-unit lengths would, thus, be due to the equivalence of acquisition and extinction schedules of exteroceptive stimulus presentation. This account not only calls into question the concept of the response unit as an integrated functional unit of behavior but also fails to provide an account of behavior in extinction situations in which such exteroceptive cues are absent.

Mowrer and Jones recognized the difficulty in interpreting extinction performance when exteroceptive stimuli which had signaled reinforcement during acquisition were no longer present. Their solution was to prevent development of such stimulus control over terminal responding by requiring that a terminal response precede reinforcement delivery. Under these conditions, they argued, the sole cue "for terminal responding consisted of kinesthetic stimuli provided by the leverpresses themselves. Whatever their source, interoceptive stimuli produced by or correlated with execution of the FR requirement provide an obvious alternative to exteroceptive sources of stimulus control. Control of terminal responding by such interoceptive response-correlated stimuli presupposes differential reinforcement of response-unit length in acquisition and could account for the maintenance of response-unit length in extinction even in the absence of obvious exteroceptive cues. It appears, in fact, that only by assuming some measure of interoceptive control over terminal responding can a discrimination model account for response-unit maintenance in extinction without vitiating the concept of the response unit as an internally cohesive response sequence.

It is possible, of course, that both interoceptive and exteroceptive stimuli exert some control over terminal responding. In the Denny et al study, however, the temporal contiguity of both sets of stimuli in extinction precluded any analysis of the relative importance of either set in controlling terminal responses. Such an analysis would be possible if exteroceptive stimulus presentation were varied independently of acquisition response-unit length. Changes in response-unit length during extinction could then be used to assess the relative importance of each stimulus type. The present experiment was designed to permit such a test by examining the effect on response-unit length and resistance to extinction produced by alteration, from acquisition to extinction, in the schedule of exteroceptive stimuli associated with completion of a ratio requirement.

\section{METHOD}

\section{Subjects}

The Ss for this experiment were 52 naive female hooded rats obtained from the breeding colony of the Department of Psychology at McMaster University. Twenty-four Ss, approximately 90-110 days old at the beginning of training, participated in Replication 1. Twenty-eight Ss, 130-150 days old at the beginning of training, participated in Replication 2. For 4 weeks prior to and throughout the study, Ss were fed a single daily ration sufficient to maintain them at $85 \%$ of ad lib weight. Session reinforcements were supplemented with Purina rat chow to complete the daily ration.

\section{Apparatus}

The apparatus consisted of four Lehigh Valley rodent test chambers enclosed in Lehigh Valley sound-insulated chests. On one wall of each chamber, a $3.75-\mathrm{cm}$ circular hole centered $3.75 \mathrm{~cm}$ above the grid floor provided access to a food tray mounted behind the wall. Entry of a rat's head into the food tray was recorded by means of a photobeam passed across the hole approximately $1.0 \mathrm{~cm}$ behind the wall. A BRS-Foringer pellet dispenser delivered $.045 \mathrm{~g}$ Noyes pellets into the food tray. Lehigh Valley retractable levers were mounted approximately $6.5 \mathrm{~cm}$ to either side of the hole, $5.0 \mathrm{~cm}$ above the floor. Only the right-hand lever, calibrated for a static weight of $22 \mathrm{~g}$, was used. Chamber illumination was provided by a $2.8-\mathrm{W}$ incandescent bulb mounted $17.5 \mathrm{~cm}$ above the food tray. Experimental control and data collection were accomplished by a Digital Equipment Corporation PDP-8/E computer.

\section{Procedure}

Rats in each replication were divided into four groups of equal size, such that the mean body weights of the groups were approximately equal. Pretraining consisted of at least four daily sessions of continuous reinforcement. During these sessions each leverpress produced two .045-g Noyes pellets and a 10-sec intertrial interval (ITI), during which the lever was retracted and the chamber darkened. Each session lasted for $75 \mathrm{~min}$, or until 30 leverpresses had occurred. For each rat continuous reinforcement continued until 10 or more leverpresses had been emit ted in each of three consecutive sessions, excluding the first. This criterion was met in the minimum of four sessions by 47 of 
the 52 Ss. One $S$ (Replication 2) required five sessions to meet criterion, three Ss (two in Replication 2 and one in Replication 1) required six sessions, and one $S$ (Replication 2) required seven sessions.

Immediately following pretraining, Ss received eight daily sessions of FR reinforcement. For half of the Ss (Groups 5-5 and 5-10) the ratio requirement was five leverpresses per reinforcement, and for the remaining Ss (Groups 10-5 and 10-10) the requirement was 10 . As in pretraining, each session consisted of 30 reinforcements, and each reinforcement was accompanied by a 10-sec ITI during which the lever was retracted and the chamber darkened.

Following FR training, Ss were extinguished in a single session to a criterion of $10 \mathrm{~min}$ without a leverpress. For half of the Ss (Groups 5-5 and 10-5) every fifth press during extinction produced a 10-sec ITI. For the remaining Ss (Groups 5-10 and 10-10) the ITI followed every tenth press. Entries into the food tray were recorded only if one or more leverpresses had occurred since the previous food tray entry.

\section{RESULTS}

Due to a photocell malfunction during extinction, the number of food tray approaches made by one $S$ in Group 10-5, Replication 2 could not be accurately determined. All data from this $\mathrm{S}$ were therefore discarded, reducing $\mathrm{N}$ in Group 10-5, Replication 2 from seven to six and overall $\mathrm{N}$ for Group 10-5 from 13 to 12 .

By the end of acquisition, control of food tray approach by stimuli associated with ratio completion was nearly complete. During the final acquisition session, no $\mathrm{S}$ made more then one such response in addition to those occurring at ratio completion. Ratio requirement and response-unit length were, therefore, virtually identical at the beginning of extinction. Response-unit lengths during extinction are presented in Table 1 in terms of mean number of leverpresses per tray approach in extinction as a function of both acquisition and extinction schedule of ITI presentation. In the absence of any statistically significant interactions between replications and either experimental variable, data from both replications were combined for this and the remaining measures.

From the response-unit length data in Table 1, it is apparent that ITI stimuli maintained considerable control over tray approach in extinction. Regardless of the FR schedule employed in acquisition, Ss extinguished on an FR 10 schedule of ITI presentation emitted significantly more leverpresses per tray approach than did Ss extinguished on an FR 5 schedule of ITI presentation $[F(1,43)=19.39, p<.001]$. There were also, however, significant differences in response-unit length as a function of acquisition FR schedule. Within each extinction condition, Ss trained on an FR 5 schedule of reinforcement made fewer leverpresses per tray approach than did Ss trained on an FR 10 schedule of reinforcement $[F(1,43)=4.84, p<.05]$. Overall, Ss in Replication 1 made significantly more leverpresses per tray approach than did $\mathrm{Ss}$ in Replication 2 $[F(1,43)=6.69, p<.05]$. As noted above, replications did not interact with either acquisition FR or extinction FR.
Table 1

Extinction Means for Response Unit Length, Food Tray Approaches, and Total Leverpresses

\begin{tabular}{lrrrr}
\hline & \multicolumn{1}{c}{$5-5$} & $5-10$ & $10-5$ & $10-10$ \\
\hline Unit Length & 5.51 & 7.27 & 6.09 & 8.83 \\
Tray Approaches & 90.00 & 69.38 & 90.25 & 76.92 \\
Leverpresses & 501.00 & 480.61 & 545.67 & 610.89 \\
\hline
\end{tabular}

With respect to the resistance of the response unit to extinction, Table 1 presents the mean number of food tray approaches emitted in extinction by each group. Contrary to the predictions of the Denny et al hypothesis, resistance of the response unit to extinction was not the same for all groups. Ss extinguished on an FR 10 schedule of ITI presentation made significantly fewer tray approaches in extinction than did Ss extinguished on an FR 5 schedule of ITI presentation $[F(1,43)=4.26, p<.05]$. In addition, the effect of extinction schedule was independent of initial training: Neither the main effect of acquisition FR nor its interaction with extinction FR approached significance. Ss in Replication 1 made significantly fewer tray approaches in extinction than did Ss in Replication 2 $[F(1,43)=4.55, p<.05]$. None of the interactions involving replications was significant.

Table 1 also presents overall resistance to extinction in terms of the mean numbers of leverpresses emitted in extinction for all combinations of acquisition and extinction FR schedules. This dependent variable is, of course, merely the product of the two that have already been presented. Overall, Ss trained on an FR 10 schedule of reinforcement tended to emit more leverpresses in extinction than did Ss trained on an FR 5 schedule of reinforcement. This difference did not, however, reach significance $[F(1,43)=2.65, p>.05]$. No other main effects or interactions approached significance.

\section{DISCUSSION}

According to a response-unit hypothesis, a sequence of responses which regularly produces reinforcement will tend to form an integrated functional unit. Evidence for such integration consists of the repetition during extinction of response sequences approximating in length those emitted during acquisition. This correspondence is presumably maintained by the discriminative control over sequence termination exercised by interoceptive stimuli correlated with completion of the previously reinforced sequence. In FR reinforcement schedules, control over terminal responding may also be exercised by exteroceptive stimuli accompanying reinforcement delivery. When such exteroceptive stimuli are also presented during extinction, interoceptive control over terminal responding, if it exists, may be extremely difficult to detect.

In the present study, evidence of such interoceptive control was obtained by introducing a discrepancy 
between interoceptive and exteroceptive cues to response-unit completion. Under these cpnditions, response-unit lengths in extinction were significantly affected by both sets of cues. Ss extinguished on an FR 10 schedule of exteroceptive stimulus presentation produced longer response sequences than did $\mathrm{Ss}$ extinguished on an FR 5 schedule of stimulus presentation. Within each extinction condition, however, Ss originally trained on an FR 5 schedule of reinforcement emitted shorter response sequences than did Ss originally trained on an FR 10 schedule of reinforcement.

The observed predominance of exteroceptive over interoceptive control may have been a result of the particular exteroceptive stimulus changes employed. The relatively long ITI used instead of a brief stimulus change may have been so salient as to overshadow interoceptive stimuli. In addition, the probability of observing a terminal response in the presence of the exteroceptive stimulus is presumably a function of its duration. The duration of the ITI may, therefore, have produced an exaggerated estimate of exteroceptive control.

The dominance of exteroceptive over interoceptive control may also have been due to differences in the difficulty of the discrimination required by each set of stimuli. Exteroceptive stimulus control required only that $\mathbf{S}$ discriminate the presence vs absence of a discrete stimulus. Interoceptive control, on the other hand, required $S$ to discriminate values of a presumably multivalued stimulus dimension whose momentary value was constantly being altered by ongoing responses.

A second purpose of the present study was to determine whether and to what extent maintenance of response-unit length from acquisition to extinction affects resistance of a response unit to extinction. In the Denny et al study, response units of different lengths showed equal resistance to extinction when response-unit lengths were maintained by appropriate schedules of exteroceptive stimulus presentation. In the present study, however, groups trained and extinguished on the same FR schedule (i.e., Groups 5-5 and 10-10) did not show equal resistance to extinction of the response unit. Regardless of initial training, Ss extinguished on an FR 10 schedule of exteroceptive stimulus presentation emitted fewer terminal responses in extinction than did Ss extinguished on an FR 5 schedule. As a direct result of this effect, the usual finding of more total leverpresses to extinction with larger FR schedules was not statistically significant in the present study. These data suggest that maintenance of equivalent response-unit lengths from acquisition to extinction is not by itself sufficient to produce equal resistance to extinction for units of different lengths.

Recently, Overmann and Denny (1973) have furnished additional evidence in support of the Denny et al findings concerning resistance of the response unit to extinction. Rats were trained to leverpress on an FR 10 schedule of reinforcement. Separate subgroups were then extinguished such that an exteroceptive stimulus associated with ratio completion (magazine click) occurred after every 6th, 10th, or 14th leverpress. As in the present study, response-unit length increased with extinction FR requirement. The number of terminal responses emitted in extinction was, however, the same for all groups.

There are two major differences between the present procedures and those used by Denny et al and Overmann and Denny which may be responsible for the discrepant results with respect to number of terminal responses to extinction as a function of response-unit length. The first of these differences concerns the criteria for terminal responses. In the present study, only those tray entries which were separated by at least one leverpress were recorded as terminal responses. In both the Denny et al and the Overmann and Denny studies, all terminal responses were recorded without regard to intervening behavior. This difference could account for the results of the present study if it were assumed that increased FR requirement is associated with an increase in the number of terminal responses that occur without intervening leverpresses. The decreased resistance to extinction shown by the FR 10 extinction groups would then be due to the failure of these additional terminal responses to be recorded. This suggestion carries the interesting implication that the strength of the response unit would be decremented by an unrewarded terminal response even though no leverpressing preceded it. Research is currently under way to evaluate this possibility.

A second procedural difference between the present study and those from Denny's laboratory is that the latter used free operant paradigms, while the present study employed discrete trials with a period of leverpress prevention following completion of each ratio requirement. It may be that independence of response-unit length and resistance of the unit to extinction does not occur in discrete-trials paradigms. A more specific possibility is that such independence occurs only when terminal responses are substantially controlled by interoceptive stimulus correlates of response-unit completion. In both the Denny et al and the Overmann and Denny studies, the exteroceptive stimulus change that was presented in extinction consisted solely of a brief click from the food magazine. In the present study, the exteroceptive stimulus was the ITI, which represented a multimodality stimulus change of substantial duration. This presumably more salient change in exteroceptive stimuli could reasonably be expected to have minimized control of terminal responding by interoceptive cues. Unfortunately, none of the available studies permits direct assessment of the role of an ITI in establishing dependence between 
response-unit length and resistance to extinction of the unit; however, studies are currently being planned to attack this problem.

\section{REFERENCES}

Boren, J. J. Resistance to extinction as a function of the fixed ratio. Journal of Experimental Psychology, 1961, 61, 305-308.

Denny, M. R., Wells, R. H., \& Maatsch, J. L. Resistance to extinction as a function of the discrimination habit established during fixed ratio reinforcement. Journal of Experimental Psychology, 1957, 54, 45 1-456.

Mowrer, O. H., \& Jones, H. M. Habit strength as a function of the pattern of reinforcement. Journal of Experimental Psychology, 1945, 35, 293-311.

Overmann, S. R., \& Denny, M. R. A free operant partial reinforcement effect: $A$ discrimination analysis. Paper presented at the Midwestern Psychological Association meeting, Chicago, May 1973.

Skinner, B. F. The behavior of organisms. New York: Appleton-Century, 1938 .

Weiss, R. F. Response speed, amplitude, and resistance to extinction as joint functions of work and length of behavior chain. Journal of Experimental Psychology, 1961, 61, 245-256.

(Received for publication October 13, 1973; accepted January 28, 1974.) 